\title{
BP-OP-1-2
}

\section{Study on the clinical characteristics of} young age pancreatic cancer using KOTUS registry

\author{
Hyung Sun KIM', Woojin KIM², Chang Soo KIM², Joon Seong PARK ${ }^{* 1}$
}

'Department of Surgery, Pancreatobiliary Cancer Clinic, Gangnam Severance Hospital, Yonsei University College of Medicine, Seoul, Korea ${ }^{2}$ Department of Preventive Medicine, Yonsei University College of Medicine, Seoul, Korea

Introduction: As the number of young patients diagnosed with pancreatic cancer increases, it is necessary to study the clinical characteristics of young pancreatic cancer patients. To date, there have been few studies of pancreatic cancer occurring at a young age. We evaluated clinical characteristics and survival rate of pancreatic cancer in young age using the KOTUS registry.

Methods: A retrospective analysis of patients of pancreatic cancer from the KOTUS registry enrolled from 1991 to 2020 . We compared demographics and clinical outcomes in patients who were younger than 45 (young age group) and older than 65 years (old age group). We estimated survival curve using the Kaplan-Meier method and hazard ratio by cox proportional hazard model.

Results: The total data was 5,617 patients, and 2,312 patients were analyzed, excluding patients with insufficient records. The old age group was 2,193 patients, and young age group was 193 patients. There were no statistically significant differences in overall survival and recurrence free survival between two groups in the total data. In the analysis of according to the stage, in Stage II-III, the rate of receiving adjuvant treatment was high in the young age group. As a result of analyzing only patients after 2016, by considering the period effect, the result of 5 -year overall survival was statistically significant lower in the young age group $(\mathrm{HR}=1.9, p=0.04)$.

Conclusions: Young age pancreatic cancer patients have the high rate of receiving adjuvant treatment, survival benefit can be obtained. Active treatment should be recommended for young patients. 